\title{
Assessment of Pathogenic Potential, Virulent Genes Profile, and Antibiotic Susceptibility of Proteus mirabilis from Urinary Tract Infection
}

\author{
Emad I. Hussein (D), ${ }^{1,2}$ Khalid Al-Batayneh, ${ }^{1}$ Majed M. Masadeh $\left(\mathbb{D},{ }^{3}\right.$ Fatina W. Dahadhah, \\ Mazhar Salim Al Zoubi, ${ }^{4}$ Alaa A. Aljabali, ${ }^{5}$ and Karem H. Alzoubi $\mathbb{D}^{6}$ \\ ${ }^{1}$ Department of Biological Sciences, Yarmouk University, Irbid 21163, Jordan \\ ${ }^{2}$ Department of Food Science and Human Nutrition, A'Sharqiyah University, Ibra, Oman \\ ${ }^{3}$ Department of Pharmaceutical Technology, Jordan University of Science and Technology, Irbid 22110, Jordan \\ ${ }^{4}$ Department of Basic Medical Sciences, Faculty of Medicine, Yarmouk University, Irbid 21163, Jordan \\ ${ }^{5}$ Department of Pharmaceutical Sciences, Yarmouk University, Irbid 21163, Jordan \\ ${ }^{6}$ Department of Clinical Pharmacy, Jordan University of Science and Technology, Irbid 22110, Jordan
}

Correspondence should be addressed to Majed M. Masadeh; mmmasadeh@yahoo.com

Received 26 September 2019; Revised 6 December 2019; Accepted 13 January 2020; Published 7 February 2020

Academic Editor: Joseph Falkinham

Copyright (c) 2020 Emad I. Hussein et al. This is an open access article distributed under the Creative Commons Attribution License, which permits unrestricted use, distribution, and reproduction in any medium, provided the original work is properly cited.

\begin{abstract}
Proteus mirabilis is the third most common bacterium that can cause complicated UTI, especially in catheterized patients. Urovirulence genes of $P$. mirabilis strains are poorly identified among UTI patients. The aims of the present study were to determine the prevalence of the uropathogenic $P$. mirabilis strains isolated from UTI patients by the detection of several $P$. mirabilis virulence genes and to characterize the antibiotic susceptibility profile of $P$. mirabilis isolates. $P$. mirabilis isolates were collected from urine specimens of patients suffering from UTI. Virulence genes in P. mirabilis, namely, $h p m A, h p m B, r s b A, l u x S$, ure C1, hlyA, rpoA, atfA, atfC, $m r p A$, and $p m 1$ were detected in the isolates via PCR detection method. All P. mirabilis virulence genes were detected in more than $90 \%$ of the isolates except $h l y A$ gene, which was detected in only $23.8 \%$ of the isolates. The rate of susceptibility for ceftriaxone was $96.8 \%$, followed by norfloxacin (82.5\%), gentamicin (71.4\%), ciprofloxacin (69.8\%), cephalexin (52.4\%), nalidixic acid (42.9\%), sulfamethoxazole (39.7\%), ampicillin (36.5\%), and nitrofurantoin (3.2\%). Significant associations $(P<0.05)$ were detected between antimicrobial susceptibility of each of the following antibiotics and the presence virulence genes. Cephalexin antimicrobial susceptibility was significantly associated with the presence each of ureC1 and atfC. Sulfamethoxazole antimicrobial susceptibility was significantly associated with the presence atfA. Ceftriaxone antimicrobial susceptibility was significantly associated with the presence each of $h p m A, \operatorname{ureC1}, r p o A$, atfC, $m r p A$, and $p m 1$. Nitrofurantoin antimicrobial susceptibility was significantly associated with the presence each of $h p m A$, ureC1, $r p o A$, atf $A$, atfC, $m r p A$, and $p m 1$. In conclusion, an association between the presence of urovirulence genes of $P$. mirabilis and increasing $P$. mirabilis resistance to antimicrobials has been demonstrated.
\end{abstract}

\section{Introduction}

Proteus mirabilis is one of the most common Gramnegative bacteria that can cause UTIs. Bacteriuria, kidney stones, catheter obstruction, acute pyelonephritis, and fever can be developed by $P$. mirabilis [1]. In fact, $P$. mirabilis strains are responsible for the majority of complicated urinary tract infections [1]. P. mirabilis is becoming resistant to antibiotics commonly used in the treatment of UTI [2]. The bacterium was shown to be highly sensitive to streptomycin (100\%), erythromycin $(85 \%)$, and sparfloxacin $(75 \%)$, whereas it showed high resistance to amoxicillin (100\%), tetracycline (95\%), and cefuroxime (80\%). Isolated Proteus mirabilis has shown 
multiple drug-resistance ability to the used antibiotics [3].

P. mirabilis encodes many virulence genes involved in infection $[4,5]$. Urease (ureC1) is a virulence gene that is important in $P$. mirabilis pathogenesis. This enzyme catalyzes the kidney and bladder stone formation or blocks indwelling urinary catheters [6]. Urease is required for urolithiasis, where it contributes in hydrolyzing urea to release ammonia, thereby increasing urinary $\mathrm{pH}$, resulting in precipitation of calcium and magnesium compounds, and urinary stone formation [7]. The alteration of $\mathrm{pH}$ is important in catheter colonization of P. mirabilis; facilitating the bacterial adherence and biofilm formation $[4,8]$. Another group of virulence genes is the quorum sensing (luxS, and $r s b A$ ). The luxS gene produces signal that is used to sense the interaction of species and its cell density in a polymicrobial community that plays critical roles in the virulence genes regulation [5]. The $r s b A$ gene expresses a histidine-containing phosphotransmitter of the bacterial two-component signaling system. This gene regulates the swarming manner, which encodes a sensory and act as a protein sensor of environmental circumstances [5]. Subsequently, $r s b A$ facilitated biofilm and extracellular polysaccharide formation [4]. The Mannose-resistant/Proteus-like fimbriae $(\mathrm{MR} / \mathrm{P})$ are related to bladder and kidney infection [9]. The $m r / p$ gene cluster comprised two transcripts: mrpABCDEFGHJ (operon) and mrpI. The main structural subunit is $m r p A$ protein, that is required at the first step of infection, including formation of clusters, and is important for wild-type levels of bladder colonization at the following steps [10]. The hemolytic activity of $P$. mirabilis is related to hemolysin $h p m A$ and $h p m B$ proteins. hpmA is mainly responsible for tissue damage, and $h p m A$ becomes active after cleavage of its $\mathrm{N}$-terminal peptide [6]. The activation and transportation of $h p m A$ depend on $h p m B$ hemolysin [6]. Previous studies suggest that hemolysin plays a critical role in UTI caused by $P$. mirabilis, which contributes to the potential urovirulence of $P$. mirabilis [11]. Another type of hemolysin proteins that Proteus species can encode and express is hlyA, and Proteus can encode $h l y A$ gene similar to that virulence gene of $E$. coli [11].

Thus, there are many virulence genes that assist survival of $P$. mirabilis within the urinary system such as urease, hemolysin, fimbriae, and flagella [12]. However, P. mirabilis strains differ in the range and expression levels of virulence genes that can affect growth of bacteria and persistence within the urinary tract. A number of studies have investigated the virulence characteristics of $P$. mirabilis and mechanisms involved in pathogenesis of UTI to identify the range of $P$. mirabilis virulence genes and their prevalence among $P$. mirabilis isolates [4]. In the present study, $P$. mirabilis isolates involving in human UTI were characterized to identify virulence gene markers in an effort to explore strategies involved in $P$. mirabilis pathogenesis and antibiotics susceptibility.

\section{Materials and Methods}

2.1. P. mirabilis Isolates. P. mirabilis isolates were collected from urine samples of patients who had UTIs and significant bacterial counts $\left(>10^{5} \mathrm{CFUs} / \mathrm{mL}\right)$ as per institutional ethics committee approval. Pure cultures were stored at $-80^{\circ} \mathrm{C}$ in Luria Bertani (LB) broth with $10 \%$ glycerol [13]. Samples were collected from July to December 2017 from Jordanian Royal Medical Services. P. mirabilis was identified as per standard diagnostic criteria using its known characteristic of swarming motility and inability to metabolize lactose on a MacConkey agar plate [8].

2.2. Antimicrobial Susceptibility Testing. The following antimicrobials were used in the current study: ciprofloxacin ( $5 \mu \mathrm{g}$, Hikma Pharmaceutical, Jordan), cephalexin (30 $\mu \mathrm{g}$, Dar Al Dawa, Jordan), nalidixic acid (30 $\mu \mathrm{g}$, Hikma Pharmaceutical, Jordan), sulfamethoxazole ( $25 \mu \mathrm{g}$, Dar Al Dawa, Jordan), ceftriaxone (30 $\mu \mathrm{g}$, Pfizer, USA), nitrofurantoin (300 $\mu \mathrm{g}$, Jordan River Pharmaceutical Industries, Amman), norfloxacin $(10 \mu \mathrm{g}$, Amman Pharmaceutical industries, Jordan), ampicillin $(10 \mu \mathrm{g}$, Jordan Veterinary and Agriculture Medical Industrial Company, Amman), and gentamicin $(10 \mu \mathrm{g}$, Hikma Pharmaceutical, Jordan).

Kirby-Bauer disk diffusion method was used to determine the susceptibility of bacteria to antibiotic agents. Bacterial colonies were transferred from the nutrient agar plate into bottles containing $\mathrm{NaCl} 0.9 \%$ to obtain bacterial density of $1.5 \times 10^{8}$ organisms per milliliter as determined by McFarland standard scale number 0.5 [14]. The cultures were uniformly streaked onto fresh Mueller Hinton agar plates using sterile cotton swabs. The plates were allowed to dry-off briefly, and then the discs of different antimicrobials were mounted onto the surface of the streaked inoculums. The plates were incubated at $37^{\circ} \mathrm{C}$ for 24 hours. Then, the culture plates were examined for inhibition. The zones of growth inhibition were measured using a meter rule described previously [15].

2.3. Extraction of Genomic DNA. For all isolates, several bacterial colonies were inoculated in $5 \mathrm{~mL}$ Luria Bertani (LB) broth media followed by incubation for 18 hours at $37^{\circ} \mathrm{C}$. $1.5 \mathrm{~mL}$ of overnight Luria broth bacterial growth culture was subjected to DNA extraction using genomic DNA isolation kit OMEGA bacterial DNA purification kit [16]. Isolated DNA samples were stored at $-20^{\circ} \mathrm{C}$ till later use.

2.4. Molecular Detection of P. mirabilis Virulence Genes. Several virulence genes were detected using conventional PCR amplification. The PCR cycling protocol and primer sequences for each gene were previously described [11, 17-20]. Confirmation of gene identity relied on finding a band corresponding to expected PCR product size.

2.5. Statistical Analysis. For the present study, statistical analysis of data using appropriate programs and methods such as the Statistical Package for the Social Sciences (SPSS) version 23 was performed to generate descriptive analysis of raw data, including generation of all frequency tables and cross tabulations. The Pearson Chi-squared test was used to 
TABle 1: Antimicrobial susceptibility of $P$. mirabilis.

\begin{tabular}{lccc}
\hline $\begin{array}{l}\text { Antimicrobial } \\
\text { agent }\end{array}$ & $\begin{array}{c}\text { Susceptible } \\
(\%)\end{array}$ & $\begin{array}{c}\text { Intermediate } \\
(\%)\end{array}$ & $\begin{array}{c}\text { Resistant } \\
(\%)\end{array}$ \\
\hline Ciprofloxacin & $44(69.8 \%)$ & $11(17.5 \%)$ & $8(12.7 \%)$ \\
Cephalexin & $33(52.4 \%)$ & $9(14.3 \%)$ & $21(33.3 \%)$ \\
Nalidixic acid & $27(42.9 \%)$ & $7(11.1 \%)$ & $29(46 \%)$ \\
Sulfamethoxazole & $25(39.7 \%)$ & $3(4.8 \%)$ & $35(55.6 \%)$ \\
Ceftriaxone & $61(96.8 \%)$ & $1(1.6 \%)$ & $1(1.6 \%)$ \\
Nitrofurantoin & $2(3.2 \%)$ & $5(7.9 \%)$ & $56(88.9 \%)$ \\
Norfloxacin & $52(82.5 \%)$ & $4(6.3 \%)$ & $7(11.1 \%)$ \\
Ampicillin & $23(36.5 \%)$ & $1(1.6 \%)$ & $39(61.9 \%)$ \\
Gentamicin & $45(71.4 \%)$ & $5(7.9 \%)$ & $13(20.6 \%)$ \\
\hline
\end{tabular}

TABLe 2: Frequency of urovirulence genes among the isolates of $P$. mirabilis.

\begin{tabular}{lc}
\hline Gene & Present $(\%)$ \\
\hline hpmA & $62(98.4 \%)$ \\
hpmB & $63(100 \%)$ \\
rsbA & $63(100 \%)$ \\
luxS & $63(100 \%)$ \\
ureC1 & $60(95.2 \%)$ \\
hlyA & $15(23.8 \%)$ \\
rpoA & $61(96.8 \%)$ \\
atfA & $62(98.4 \%)$ \\
atfC & $60(95.2 \%)$ \\
mrpA & $58(92.1 \%)$ \\
pm1 & $58(92.1 \%)$ \\
\hline
\end{tabular}

compare frequency data. $P$ value less than 0.05 was considered statistically significant.

\section{Results}

3.1. Antimicrobial Susceptibility Results. The antimicrobial susceptibility results of the $P$. mirabilis isolates to several antimicrobial agents are shown in Table 1, which represents results as susceptible, intermediate, and resistant. The rate of antibiotic resistance was highest for nitrofurantoin $(88.9 \%)$, whereas it was lowest for ceftriaxone (1.6\%).

3.2. P. mirabilis Urovirulence Genes. Detected rates of virulence genes are shown in Table 2. The antimicrobial susceptibility was highly correlated with the presence of $P$. mirabilis virulence genes (Table 3). Moreover, $h p m A$, ureC1, $r p o A$, atfC, $m r p A$, and $p m 1$ urovirulence genes were more likely to coexist with each other at $P$. mirabilis $(P<0.001)$.

\section{Discussion}

In the current study, $P$. mirabilis isolated from UTI patients were analyzed for the presence of virulence genes and susceptibility to antimicrobials. $P$. mirabilis genes associated with UTIs may be valuable in developing strategies for treating and preventing UTIs. The results of this study provide evidence supporting the role of urovirulence genes of $P$. mirabilis in human UTIs.

Current results showed the association between resistance to certain antibiotics and the presence of $P$. mirabilis urovirulence genes. For example, ure $\mathrm{Cl}$ and atfC genes were associated with resistance to cephalexin, atfA with resistance to sulfamethoxazole, $h p m A$, ureC1, rpoA, atfC, $m r p A$, and $p m 1$ with resistance to ceftriaxone, and $h p m A, u r e C 1, r p o A$, atf $A$, atfC, $m r p A$, and $p m 1$ with resistance to nitrofurantoin.

The correlation between presence of these genes and the increase in resistance toward antibiotics may be attributed to pathogenicity of these genes and their functional roles such as urease, hemolysins, and fimbriae that help the organism to overcome host defense mechanisms and colonize the urinary tract. Overall, these results explain the potential of these uropathogens to interfere with the infection treatment, impair the action of host immune cells, and weaken the antibiotic efficiency.

Most isolates were resistant to nitrofurantoin (88.9\%), ampicillin (61.9\%), and sulfamethoxazole (55.6\%). On the contrary, the highest sensitivity was against ceftriaxone (96.8\%), norfloxacin (82.5\%), gentamicin (71.4\%), and ciprofloxacin $(69.8 \%)$. Similar results have been reported for $P$. mirabilis from Nigeria, where isolates' resistance rates to ciprofloxacin, nalidixic acid, sulfamethoxazole, and gentamicin were $13.9 \%, 53.7 \%, 74.1 \%$, and $26.9 \%$, respectively [21]. In Czech Republic, the isolates had a resistance rate of ciprofloxacin (35.2\%), sulfamethoxazole (39.0\%), ampicillin (38.5\%), and gentamicin (25.4\%), which are different form findings of the present study [22]. The noticed variations in resistance rates may be referred to regional variation in bacterial strain and virulence genes prevalence, in addition to different standards and controls for prescription and use of antimicrobial agents.

Urovirulence genes of $P$. mirabilis strains are poorly identified among UTI patients. One of the aims of this study was to identify the urovirulence genes of $P$. mirabilis strains isolated from UTI symptomatic patients. Specifically, we investigated the presence of urovirulence genes $h p m A$, $h p m B, r s b A, l u x S$, ureC1, hlyA, rpoA, atfA, atfC, $m r p A$, and $p m 1$ using PCR-based analysis. Certain patterns of virulence genes and distributions were identified among the isolates. Statistically significant associations were observed among the $P$. mirabilis urovirulence genes, as some genes were more likely to coexist with other genes. There was coassociation between $h p m A$, ureC1, rpoA, atfC, $m r p A$, and $p m 1$ genes. Therefore, it is likely that a frequent occurrence of antimicrobial resistance is due to the presence of multiple resistance genes that increase the $P$. mirabilis pathogenicity.

Virulence genes were detected at the following rates among the isolates: $h p m B, r s b A$, and $l u x S$ at $100 \%, h p m A$ and atf $A$ at $98.4 \%, r p o A$ at $96.8 \%$, ure $C 1$ and atfC at $95.2 \%$, $m r p A$ and $p m 1$ at $92.1 \%$, and $h l y A$ at $23.8 \%$. Some of these prevalence rates are different from those reported from other countries $[4,11,22]$. Prevalence of these genes may vary according to the clinical status of the host and the genetic makeup of the isolates causing UTIs. The $h p m B$, luxS, and $r s b A$ genes were the most prevalent at $100 \%$, followed by $h p m A$ and atfA at $98.4 \%$ each, while the $h l y A$ gene was the least prevalent at $23.8 \%$. Other urovirulence genes were prevalent in $92-97 \%$ of the isolates. Additionally, the high prevalence of $h p m B$ and $h p m A$ at $100 \%$ and $98.4 \%$, respectively, in the present study was 
TABLe 3: Association between urovirulence genes and susceptibility to antimicrobial agents in P. mirabilis isolates.

\begin{tabular}{|c|c|c|c|c|c|c|c|c|c|c|c|c|c|c|c|c|c|c|c|c|c|c|c|c|c|c|c|c|}
\hline \multicolumn{2}{|c|}{$\begin{array}{l}\text { Antibiotics/ } \\
\text { genes }\end{array}$} & \multicolumn{3}{|c|}{ CIP } & \multicolumn{3}{|c|}{ CL } & \multicolumn{3}{|c|}{ NA } & \multicolumn{3}{|c|}{ SMX } & \multicolumn{3}{|c|}{ CTX } & \multicolumn{3}{|c|}{ NFT } & \multicolumn{3}{|c|}{ NFX } & \multicolumn{3}{|c|}{ AMP } & \multicolumn{3}{|c|}{ GN } \\
\hline \multirow{4}{*}{ hpmA } & & $S$ & I & $\mathrm{R}$ & $S$ & I & $\mathrm{R}$ & $S$ & I & $\mathrm{R}$ & $\mathrm{S}$ & I & $\mathrm{R}$ & $S$ & I & $\mathrm{R}$ & S & I & $\mathrm{R}$ & $\mathrm{S}$ & I & $\mathrm{R}$ & S & I & $\mathrm{R}$ & $S$ & I & $\overline{\mathrm{R}}$ \\
\hline & - & 1 & 0 & 0 & 0 & 0 & 1 & 0 & . & 1 & 0 & 0 & 1 & 0 & 0 & 1 & 1 & 0 & 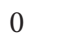 & 1 & 0 & 0 & 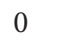 & 0 & & 1 & 0 & 0 \\
\hline & + & 43 & 11 & 8 & 33 & 92 & 20 & 27 & 7 & 28 & 25 & 33 & 34 & 61 & 1 & 0 & 1 & $5 \quad 5$ & 56 & 51 & 4 & 7 & 23 & 13 & 38 & 44 & 5 & 13 \\
\hline & $P$ value & \multicolumn{3}{|c|}{0.803} & \multicolumn{3}{|c|}{0.362} & \multicolumn{3}{|c|}{0.551} & \multicolumn{3}{|c|}{0.666} & \multicolumn{3}{|c|}{0.000} & \multicolumn{3}{|c|}{0.000} & & 898 & & & 732 & & & .816 & \\
\hline & - & 0 & 0 & 0 & 0 & 0 & 0 & 0 & 0 & 0 & 0 & 0 & 0 & 0 & 0 & 0 & 0 & 0 & 0 & 0 & 0 & 0 & 0 & 0 & 0 & 0 & 0 & 0 \\
\hline$h p m B$ & + & 44 & 11 & 8 & 33 & 92 & 21 & 27 & 7 & 29 & 25 & 33 & 35 & 61 & 1 & 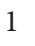 & 2 & 55 & 56 & 52 & 4 & 7 & 23 & 13 & 39 & 45 & 5 & 13 \\
\hline & $P$ value & & - & & & - & & & - & & & - & & & - & & & - & & & - & & & - & & & - & \\
\hline & - & 0 & 0 & 0 & 0 & 0 & 0 & 0 & 0 & 0 & 0 & 0 & 0 & 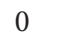 & 0 & 0 & v & 0 & 0 & 0 & 0 & 0 & U & 0 & 0 & 0 & 0 & 0 \\
\hline$r s b A$ & + & 44 & 11 & 8 & 33 & 92 & 21 & 27 & 7 & 29 & 25 & 33 & 35 & 61 & 1 & & 2 & $5 \quad 5$ & 56 & 52 & 4 & 7 & 23 & 13 & 39 & 45 & 5 & 13 \\
\hline & $P$ value & & - & & & - & & & - & & & - & & & - & & & - & & & - & & & - & & & - & \\
\hline & - & 0 & 0 & 0 & 0 & 0 & 0 & 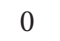 & 0 & 0 & 0 & 0 & 0 & 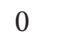 & 0 & 0 & 0 & 0 & 0 & 0 & 0 & 0 & 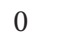 & 0 & 0 & 0 & 0 & 0 \\
\hline$i x S$ & + & 44 & 11 & 8 & 33 & 92 & 21 & 27 & 7 & 29 & 25 & 33 & 35 & 61 & 1 & & 2 & $5 \quad 5$ & 56 & 52 & 4 & 7 & 23 & 13 & 39 & 45 & 5 & 13 \\
\hline & $P$ value & & - & & & - & & & - & & & - & & & - & & & - & & & - & & & - & & & - & \\
\hline & - & 1 & 2 & 0 & 0 & 0 & 3 & 0 & 0 & 3 & 1 & 0 & 2 & 2 & 0 & 1 & 1 & 2 & 0 & 3 & 0 & 0 & 0 & 0 & 3 & 3 & 0 & 0 \\
\hline$e C 1$ & 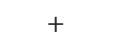 & 43 & 9 & 8 & 33 & $\begin{array}{ll}9 & 1\end{array}$ & 18 & 27 & , & 26 & 24 & 33 & 3 & 59 & 1 & c & 1 & 35 & 56 & 49 & 4 & 7 & 23 & 13 & 36 & 42 & 5 & 13 \\
\hline & $P$ value & & .068 & & & .043 & & & .158 & & & .882 & & & 000 & & & 0.000 & & & 717 & & & 379 & & & .533 & \\
\hline & - & 34 & 9 & 5 & 22 & $\begin{array}{ll}9 & 1\end{array}$ & 17 & 18 & 6 & 24 & 18 & 22 & 28 & 47 & 0 & 1 & 2 & 44 & 42 & 39 & 4 & 5 & 18 & 12 & 29 & 33 & 4 & 11 \\
\hline hlyA & + & 10 & 2 & 3 & 11 & 0 & 4 & 9 & 1 & 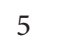 & 7 & 1 & 7 & 14 & 1 & & 0 & 11 & 14 & 13 & 0 & 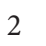 & - & $0 \quad 1$ & 10 & 12 & 1 & 2 \\
\hline & $P$ value & & .592 & & & .094 & & & .303 & & & .715 & & & 171 & & & 0.702 & & & 502 & & & 803 & & & .687 & \\
\hline & - & 1 & 1 & 0 & 0 & 0 & 2 & 0 & 0 & 2 & 1 & 0 & 1 & 1 & 0 & 1 & 1 & 1 & 0 & 2 & 0 & 0 & 0 & 0 & 2 & 2 & 0 & 0 \\
\hline rpoA & + & 43 & 10 & 8 & 33 & $\begin{array}{ll}9 & 1\end{array}$ & 19 & 27 & 7 & 27 & 24 & 33 & 34 & 60 & 1 & 0 & 1 & 45 & 56 & 50 & 4 & 7 & 23 & 13 & 37 & 43 & 5 & 13 \\
\hline & $P$ valu & & .442 & & & .127 & & & .298 & & & .921 & & & 000 & & & 0.000 & & & 804 & & & 530 & & & .662 & \\
\hline & - & 1 & 0 & 0 & 0 & 0 & 1 & 0 & 0 & . & 0 & 1 & 0 & 1 & 0 & 0 & 1 & 0 & 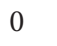 & 1 & 0 & 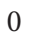 & 0 & 0 & . & 1 & 0 & 0 \\
\hline $\operatorname{atfA}$ & + & 43 & 11 & 8 & 33 & 92 & 20 & 26 & 7 & 29 & 25 & 23 & 35 & 60 & 1 & 1 & 1 & $5 \quad 5$ & 56 & 51 & 4 & 7 & 23 & 13 & 38 & 44 & 5 & 13 \\
\hline & $P$ valu & & .803 & & & .362 & & & .508 & & & .000 & & & 983 & & & 0.000 & & & 898 & & & 732 & & & .816 & \\
\hline & - & 1 & 2 & 0 & 0 & 0 & 3 & 0 & 0 & 3 & 1 & 0 & 2 & 2 & 0 & 1 & 1 & 2 & 0 & 3 & 0 & 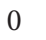 & 0 & 0 & 3 & 3 & 0 & 0 \\
\hline atfC & 1 & 43 & 9 & 8 & 33 & $\begin{array}{ll}9 & 1\end{array}$ & 18 & 27 & 7 & 26 & 24 & 33 & 33 & 59 & 1 & 0 & 1 & 35 & 56 & 49 & 4 & 7 & 23 & 13 & 36 & 42 & 5 & 13 \\
\hline & $P$ value & & .068 & & & .043 & & & .158 & & & .882 & & & 000 & & & 0.000 & & & 717 & & & 379 & & & .533 & \\
\hline & - & 3 & 1 & 1 & 1 & 1 & 3 & 0 & 1 & 1 & 2 & 1 & 2 & 4 & 0 & 1 & 1 & 1 & 3 & 4 & 0 & 1 & 1 & 0 & 4 & 4 & 0 & 1 \\
\hline$m r p A$ & + & 41 & 10 & 7 & 32 & $\begin{array}{ll}8 & 1\end{array}$ & 18 & 27 & 6 & 25 & 23 & 23 & 33 & 57 & 1 & 0 & 1 & 45 & 53 & 48 & 4 & 6 & 22 & 13 & 35 & 41 & 5 & 12 \\
\hline & $P$ value & & .851 & & & .306 & & & .130 & & & .236 & & & 003 & & & 0.042 & & & 692 & & & 677 & & & .784 & \\
\hline & - & 2 & 2 & 1 & 2 & 2 & 1 & 0 & 1 & 4 & 2 & 0 & 3 & 4 & 0 & 1 & 1 & 13 & 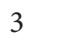 & 4 & 0 & 1 & 2 & 0 & 3 & 4 & 0 & 1 \\
\hline$p m 1$ & + & 42 & 9 & 7 & 31 & $8 \quad 1$ & 19 & 27 & 6 & 25 & 23 & 33 & 32 & 57 & 1 & 0 & 1 & 45 & 53 & 48 & 4 & 6 & 21 & 13 & 36 & 41 & 5 & 12 \\
\hline & $P$ value & & .286 & & & .837 & & & .130 & & & .870 & & & 003 & & & 0.042 & & & 692 & & & 948 & & & .784 & \\
\hline
\end{tabular}

Ciprofloxacin: CIP, cephalexin: CL, nalidixic acid: NA, sulfamethoxazole: SMX, ceftriaxone: CTX, nitrofurantoin: NFT, norfloxacin: NFX, ampicillin: AMP, and gentamicin: GN.

consistent with a previous report from Brazil [11]. On the contrary, the prevalence of $h l y A(23.8 \%)$ is different from the same study, which confirmed that none of the isolates presented hly $A$ gene [11]. Interestingly, another study from Iraq reported ureC1, $m r p A, p m 1$, luxS, and $r s b A$ prevalence rates of $18 \%, 35 \%, 41 \%, 47 \%$, and $53 \%$, respectively, which are not comparable to our findings [4], whereas a study from Iran reported $l u x S$, and $r s b A$ prevalence rate of $70 \%$ each [17]. The previously mentioned prevalence rates most likely attribute to the differences in the distribution of virulence genes among different populations and geographic locations. The current study has some limitations including that it tested only certain virulence genes and certain antibiotics. Studying more virulence genes and antibiotics is recommended future study.

None of the previous studies have investigated a role of atfA and atfC urovirulence genes in P. mirabilis UTIs. Current results suggest that ATF fimbriae could have an important role in adhesion and biofilm formation on abiotic $[20,23]$.

In conclusion, $P$. mirabilis isolates demonstrated high susceptibility against ceftriaxone, norfloxacin, gentamicin, and ciprofloxacin, and high resistance against nitrofurantoin, ampicillin, and sulfamethoxazole. In addition, significant associations between virulence genes and resistance phenotypes were identified, which suggests increased resistance to antimicrobial agents due to the presence of these virulence genes.

\section{Data Availability}

The data used to support the findings of this study are available from the corresponding author upon request.

\section{Conflicts of Interest}

The authors declare that they have no conflicts of interest. 


\section{Acknowledgments}

This project was funded by Deanship of Research at Jordan University of Science and Technology.

\section{References}

[1] J. N. Schaffer and M. M. Pearson, "Proteus mirabilis and urinary tract infections," Microbiology Spectrum, vol. 3, 2015.

[2] R. T. Jamil, L. A. Foris, and J. Snowden, "Proteus mirabilis infections," StatPearls, Treasure Island, FL, USA, 2019.

[3] M. Umar, A. Arzai, G. Yusuf et al., "Serological characterization and antimicrobial sensitivity profile of Haemophilus influenzae serotypes isolated from aminu kano teaching hospital, kano, Nigeria," British Microbiology Research Journal, vol. 15, no. 5, pp. 1-10, 2016.

[4] K. F. Abbas, J. K. Al Khafaji, and M. S. Al-Shukri, "Molecular detection of some virulence genes in Proteus mirabilis isolated from hillaprovince," International Journal of Research Studies in Biosciences, vol. 3, pp. 85-89, 2015.

[5] R. M. Morgenstein, B. Szostek, and P. N. Rather, "Regulation of gene expression during swarmer cell differentiation in Proteus mirabilis," FEMS Microbiology Reviews, vol. 34, no. 5, pp. 753-763, 2010.

[6] C. E. Armbruster, H. L. T. Mobley, and M. M. Pearson, "Pathogenesis of Proteus mirabilis infection," EcoSal Plus, vol. 8, no. 1, 2018.

[7] C. E. Armbruster and H. L. T. Mobley, "Merging mythology and morphology: the multifaceted lifestyle of Proteus mirabilis," Nature Reviews Microbiology, vol. 10, no. 11, pp. 743-754, 2012.

[8] J. N. Schaffer, A. N. Norsworthy, T.-T. Sun, and M. M. Pearson, "Proteus mirabilis fimbriae-and urease-dependent clusters assemble in an extracellular niche to initiate bladder stone formation," Proceedings of the National Academy of Sciences, vol. 113, no. 16, pp. 4494-4499, 2016.

[9] R. Pellegrino, U. Galvalisi, P. Scavone, V. Sosa, and P. Zunino, "Evaluation of Proteus mirabilis structural fimbrial proteins as antigens against urinary tract infections," FEMS Immunology \& Medical Microbiology, vol. 36, no. 1-2, pp. 103-110, 2003.

[10] A. N. Norsworthy and M. M. Pearson, "From catheter to kidney stone: the uropathogenic lifestyle of Proteus mirabilis," Trends in Microbiology, vol. 25, no. 4, pp. 304-315, 2017.

[11] S. E. Cestari, M. S. Ludovico, F. H. Martins, S. P. D. da Rocha, W. P. Elias, and J. S. Pelayo, "Molecular detection of HpmA and HlyA hemolysin of uropathogenic Proteus mirabilis," Current Microbiology, vol. 67, no. 6, pp. 703-707, 2013.

[12] L. S. Burall, J. M. Harro, X. Li et al., "Proteus mirabilis genes that contribute to pathogenesis of urinary tract infection: identification of 25 signature-tagged mutants attenuated at least 100-fold," Infection and Immunity, vol. 72, no. 5, pp. 2922-2938, 2004.

[13] S. L. Chiang and E. J. Rubin, "Construction of a mariner-based transposon for epitope-tagging and genomic targeting," Gene, vol. 296, no. 1-2, pp. 179-185, 2002.

[14] S. Irfan, A. Zafar, D. Guhar, T. Ahsan, and R. Hasan, "Metallo$\beta$-lactamase-producing clinical isolates of Acinetobacter species and Pseudomonas aeruginosa from intensive care unit patients of a tertiary care hospital," Indian Journal of Medical Microbiology, vol. 26, no. 3, pp. 243-245, 2008.

[15] M. Umar, D. Akafyi, Y. Jobbi, A. Ayaya, and I. Abdulkarim, "Biochemical characterization and antibiogram pattern of Streptococcus mutans isolated from Dental Unit, Sick-Bay, Ahmadu Bello University, Zaria, Nigeria," International
Journal of Biological and Biomedical Sciences, vol. 4, pp. 63-66, 2015.

[16] S. Aghamiri, N. Amirmozafari, J. Fallah, B. Fouladtan, and H. Kafil, "Antibiotic resistance pattern and evaluation of metallo-beta lactamase genes including bla-IMP and bla-VIM types in Pseudomonas aeruginosa isolated from patients in Tehran Hospitals," ISRN Microbiology, Article ID 941507, 6 pages, 2014.

[17] S. A. Badi, J. Norouzy, and A. A. Sepahi, "Detection Rsba gene's band \& effect of miristic acid in virulence of Proteus mirabilis isolated from urinary tract infrction," Iranian Journal of Public Health, vol. 43, p. 210, 2014.

[18] H. S. Huang, J. Chen, L. J. Teng, and M. K. Lai, "Use of polymerase chain reaction to detect Proteus mirabilis and Ureaplasma urealyticum in urinary calculi," Journal of the Formosan Medical Association, vol. 98, no. 98, pp. 844-850, 1999.

[19] V. Sosa, G. Schlapp, and P. Zunino, "Proteus mirabilis isolates of different origins do not show correlation with virulence attributes and can colonize the urinary tract of mice," $M i$ crobiology, vol. 152, no. 7, pp. 2149-2157, 2006.

[20] P. Zunino, L. Geymonat, A. G. Allen, C. Legnani-Fajardo, and D. J. Maskell, "Virulence of a Proteus mirabilis ATF isogenic mutant is not impaired in a mouse model of ascending urinary tract infection," FEMS Immunology \& Medical Microbiology, vol. 29, no. 2, pp. 137-143, 2000.

[21] O. S. Alabi, N. Mendonça, O. E. Adeleke, and G. J. da Silva, "Molecular screening of antibiotic-resistant determinants among multidrug-resistant clinical isolates of Proteus mirabilis from SouthWest Nigeria," African Health Sciences, vol. 17, no. 2, pp. 356-365, 2017.

[22] L. Cernohorska and E. Chvilova, "Proteus mirabilis isolated from urine, resistance to antibiotics and biofilm formation," Klinicka Mikrobiologie a Infekcni Lekarstvi, vol. 17, pp. 81-85, 2011.

[23] P. Scavone, V. Iribarnegaray, A. L. Caetano, G. Schlapp, S. Hartel, and P. Zunino, "Fimbriae have distinguishable roles in Proteus mirabilis biofilm formation," Pathogens and Disease, vol. 74, 2016. 